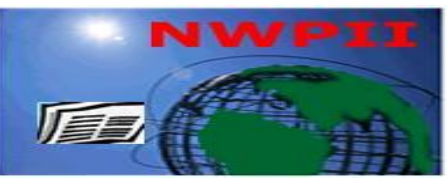

American Journal of Biomedical Sciences

ISSN: 1937-9080

nwpii.com/ajbms

\title{
Comparing Trio-Modified Papanicolaou Staining Methods for Assessing Liquid-Based Cytology Samples
}

\section{Bolaji Efosa ODIGIE*1, 2,3 and Peter Uwadiegwu ACHUKWU ${ }^{2,3}$}

${ }^{1}$ Medical Laboratory Science, School of Basic Medical Sciences, College of Medical Sciences, University of Benin, Benin City, Nigeria.

${ }^{2}$ Medical Laboratory Science, Faculty of Health Sciences and Technology, College of Medicine, University of Nigeria, Enugu Campus (UNEC), Enugu, Nigeria.

${ }^{3}$ Sexual Health Concerns for Commercial Sex Workers (SHCCSW), University of Benin, Benin City, Nigeria.

"Corresponding Author

Bolaji Efosa ODIGIE

Medical Laboratory Science

School of Basic Medical Sciences

College of Medical Sciences

University of Benin

Benin City

Nigeria

Mobile: +2348023345132

Email: bolaji.odigie@uniben.edu

Received: 17 May 2017; | Revised: 14 July 2017; | Accepted: 31 July 2017

\begin{abstract}
The present study compared the staining uptake of three modified Papanicolaou staining methods in the assessment of liquid-based cytology samples. Other goals were to check for the most desirable and outstanding qualitative staining method amongst the three modified techniques. Six hundred and sixty (660) liquid-based cytology (LBC) smear samples were collected from 220 patients (3 slides per patient) attending the Center for Disease and Infection Control (CDC), University of Benin Teaching Hospital between January 2014 and April 2016. The LBC smears were marked (L1 to L220) for the Richard Allan Thermoscientific (RAT) method with the corresponding (M1 to M220) for an earlier modified emergency method (MEM) and (N1 to N220) marked to demonstrate the Simplified Alternative Papanicolaou (SAP) method comparatively. SAP lasted for $(5 \pm 0.5)$ minutes compared to MEM (7 \pm 0.5$)$ and RAT $(25 \pm 0.5)$ minutes for the same number of slides $(\mathrm{P}<0.001)$. Also, RAT had 3/220(1.4\%), MEM 0/220(0\%) and SAP 3/220(1.4\%) unsatisfactory cytoplasmic staining. The nuclei staining was optimal and distinct in RAT 1/220(0.5\%), MEM 2/220(0.9\%) and SAP 3/220(1.4\%) for the same number of stained smears $(\mathrm{P}<0.00)$. Our findings indicate that SAP is both time-saving and economic friendly than other two methods. However, MEM showed an excellent qualitative staining for LBC samples than SAP and RAT.
\end{abstract}


Keywords: Liquid-based cytology, conventional Papanicolaou staining, modified staining method and Cytodiagnostics.

\section{Introduction}

The liquid-based cytology (LBC) is currently the method of choice for the collection of Pap smears, with the conventional Pap staining method experiencing different forms of modification to suit the modern day diagnoses. LBC offers an advantage over the conventional smears for women who have excessive cervical mucus, discharge or blood. LBC leads to significantly fewer unsatisfactory smears with related financial costs and a reduced apprehension for women. The acceptable staining technique for cytology is the Papanicolaou method and has undergone several modifications over the years ${ }^{[1]}$. These modifications are aimed at either to make the stained product better or to correct any unforeseen and existing deficiency in the technique ${ }^{[2]}$ and by achieving a more desirable method that is capable of diagnosing cancer without stress and minimum time utilized.

Papanicolaou technique employs three major stains used as a conventional procedure: Harris hematoxylin, Orange G6 and Eosin-azure 50 or 56 for cervical cytology (Pap smear), fine needle aspiration cytology (FNAC) and the liquid-based cytology (LBC) screening ${ }^{[1]}$. The search for a staining procedure that is suitable for all purposes is of great importance to developing countries and economically depressed- developing nations; where cost effectiveness and a fast turn-around time are major concerns. Moreover, recall that the conventional Pap stain initially developed by George Papanicolaou in 1942, has a polychromatic and transparent staining ability that crisps the nuclear and cytoplasmic materials and is the basis to which cytological diagnosis is made ${ }^{[3]}$. Briefly, the conventional stain by George Papanicolaou possesses the cytoplasmic counterstains for all gynaecological specimens, which encompass natural dyes and reagent solvents used in traditional Eosin-Azure formulations. Also, cellular results are consistent as well with basal or intermediate and surface cell stainings. The multicolour effect from the conventional method is usually due to the discerning stain uptake by the component cells and their affinities for specific dyes ${ }^{[1,4]}$. The various formulations provide the end users with control over colour intensities and the hues ability of the counterstains. However, the procedure though has the widest use in cytology diagnosis but not without some shortcomings especially on routine use. One of which is the large scale use of alcohol in the staining procedure as a dehydrating agent. Others are the huge cost implication and limitation in the rigorous purchasing license as well as the doubtful originality of the products ${ }^{[4,5]}$.

The staining characteristics of the conventional Pap staining method has stood the test of time, however, intended modified Pap staining procedures for routine cytological screening, should have an excellent staining ability ${ }^{[6,7]}$. Also, in the conceptual technique, it is expected that speed and accuracy of the procedure, as well as the cost effectiveness of overall improved services to the patients, are envisaged ${ }^{[6-9]}$. Again, the anticipated technique should not be time-consuming and must be able to explicitly define the morphological attributes of the cytology specimens on examination ${ }^{[9]}$. By the preceding, therefore, this study was to compare the resultant staining uptake of triomodified Papanicolaou staining methods (three modification procedures) in the assessment of LBC samples. Other goals were to refer the most desirable and outstanding qualitative staining method amongst the three modified techniques suitable for use in Cytopathology laboratories with a quick turn-around time, cutting cost and retaining the enhanced staining abilities. The basis of compares will center on the stains uptake, quality staining contrast, hues and intensities in addition to the cost of production and time allowed for a whole batch of stained smears.

\section{Methods}

\subsection{Ethics Clearance}

(C) 2017 by NWPII. All rights reserved 
Ethical approval was obtained from University of Benin Teaching Hospital (UBTH), Benin City, Nigeria (Protocol no: ADM/E22/A/VOL. VII/951).

\subsection{Staining Technique}

Papanicolaou staining has been in use for decades and has been published to date ${ }^{[1,3-10]}$. This study was a follow-up to an existing work and an adaptation of the study by Odigie and Obaseki ${ }^{[1]}$.

In the former, the modified emergency method (MEM) was tested on Pap and fine needle aspiration cytology smears and by comparing with the conventional Papanicolaou technique (CPT). In this study, six hundred and sixty (660) liquid-based cytology smears from 220 patients were collected (3 slides per patient) from the Center for Disease and Infection Control (CDC), Community Health Department, UBTH between January 2014 and April 2016. LBC smears were marked (L1 to L220) for the Richard Allan Thermo scientific (RAT) method with the corresponding (M1 to M220) for an earlier modified emergency method (MEM) and (N1 to N220) marked to demonstrate the Simplified Alternative Papanicolaou (SAP) method comparatively. A detailed RAT staining method by Richard ${ }^{[5]}$ and MEM by Odigie and Obaseki ${ }^{[1]}$ have been published in reputable journals and are since put into use in various cytodiagnostic laboratories in Nigeria. In the present study, the simplified alternative Papanicolaou (SAP) method is a further modification of the modified emergency method (MEM), which might also be seen as a remodified emergency approach. In the SAP method, the alcohol grades were replaced by a single $1 \%$ glacial acetic acid. Differentiation and dehydration processes were excluded. Harris hematoxylin for nuclei uptake stained for $1 \mathrm{~min} 30 \mathrm{sec}$ but preheated to $60^{\circ} \mathrm{C}$ before use. Preheat was carried out in a hot air oven before staining for rapid penetration. All alcohol stages were replaced with $1 \%$ glacier acetic acid except for the final dehydration process using a single batch of absolute alcohol for 1 minute.

Table 1: Summary of the methods used for LBC staining

\begin{tabular}{|c|c|c|}
\hline Richard's Method & Modified Emergency Method & Simplified Alternative Pap Method \\
\hline $\begin{array}{c}\text { Hydrate with } \\
\text { descending grades of } \\
\text { alcohol }\end{array}$ & Use $0.5 \%$ glacier acetic acid & Use $1 \%$ glacier acetic acid \\
\hline $\begin{array}{l}\text { Harris haematoxylin } \\
\quad(4-10 \mathrm{mins})\end{array}$ & $\begin{array}{c}45^{0 \mathrm{c}} \text { pre-heat Harris } \\
\text { haematoxylin }(2 \mathrm{mins})\end{array}$ & $60^{0 \mathrm{c}}$ pre-heat Harris haematoxylin (1min) \\
\hline $\begin{array}{l}\text { Rinse with distilled } \\
\text { water }\end{array}$ & Rinse with water & Rinse with water \\
\hline $\begin{array}{l}\text { Differentiate with } 1 \% \\
\text { acid alcohol }\end{array}$ & Skipped & Skipped \\
\hline $\begin{array}{l}\text { Rinse with distilled } \\
\text { water }\end{array}$ & Skipped & Skipped \\
\hline $\begin{array}{l}\text { Blue in running tap } \\
\text { water (10minutes) }\end{array}$ & Skipped & Skipped \\
\hline $\begin{array}{c}\text { Rinse two changes of } \\
95 \% \text { alcohol } \\
(2 \text { minutes each })\end{array}$ & $\begin{array}{l}\text { Rinse Use } 0.5 \% \text { glacier acetic } \\
\text { acid ( } 15 \text { seconds) }\end{array}$ & $\begin{array}{l}\text { Rinse } 1 \% \text { glacier acetic acid } \\
\qquad(10 \text { seconds })\end{array}$ \\
\hline
\end{tabular}




\begin{tabular}{|c|c|c|}
\hline $\begin{array}{l}\text { Stain in Orange G6 } \\
(2 \text { minutes })\end{array}$ & $\begin{array}{l}\text { Stain in OG6 + EA50 pre- } \\
\text { mixed in equal proportion } \\
\text { (1minute and } 45 \text { seconds) }\end{array}$ & $\begin{array}{l}\text { Stain in OG6 + EA50 pre-mixed in equal } \\
\text { proportion (1 minute and 30seconds) }\end{array}$ \\
\hline $\begin{array}{l}\text { Rinse } 2 \text { changes of } \\
95 \% \text { alcohol } \\
\text { (2minutes each) }\end{array}$ & $\begin{array}{l}\text { Rinse Use } 0.5 \% \text { glacier acetic } \\
\text { acid ( } 15 \text { seconds })\end{array}$ & $\begin{array}{c}\text { Rinse Use } 0.5 \% \text { glacier acetic acid (10 } \\
\text { seconds) }\end{array}$ \\
\hline $\begin{array}{l}\text { Stain in Eosin-azure } \\
\text { (EA 50) for } 2 \text { minutes }\end{array}$ & Skipped & Skipped \\
\hline $\begin{array}{l}\text { Rinse two changes of } \\
95 \% \text { alcohol } \\
\text { (2minutes each) }\end{array}$ & Skipped & Skipped \\
\hline $\begin{array}{c}\text { Dehydrate in } 2 \\
\text { changes of absolute } \\
\text { alcohol ( } 2 \text { minutes } \\
\text { each) }\end{array}$ & $\begin{array}{l}\text { A single bath of absolute } \\
\text { alcohol for 1minute }\end{array}$ & $\begin{array}{l}\text { A single bath of absolute alcohol for } \\
\text { 1minute }\end{array}$ \\
\hline Clear in Xylene & Clear in Xylene & Clear in Xylene \\
\hline Mount in DPX & Mount in DPX & Mount in DPX \\
\hline $\begin{array}{l}\text { Approximate time } \\
\text { used } \\
\text { (25 minutes) }\end{array}$ & $\begin{array}{l}\text { Approximate time used } \\
\quad \text { (7 minutes) }\end{array}$ & $\begin{array}{l}\text { Approximate time used } \\
\quad(5 \text { minutes })\end{array}$ \\
\hline Richard $^{[5]}$ & Odigie and Obaseki ${ }^{[1]}$ & Present study \\
\hline
\end{tabular}

\subsection{Statistical Analyses}

Data was presented in percentages where necessary. A Chi-square test was calculated to check the importance of the data from the triomethods using Minitab statistical software version 16.0 , and the significance was set at $\mathrm{p}<0.05$.

\section{Results}

The present study showed an overall considerable time engaged in staining an LBC slide or bunch of slides in SAP (5 \pm 0.5$)$ minutes compared to MEM ( $7 \pm 0.5)$ and RAT $(25 \pm 0.5)$ minutes for the same number of slides $(\mathrm{P}<0.001)$. Also, RAT had 3/220(1.4\%), MEM 0/220(0\%) and SAP $3 / 220(1.4 \%)$ unsatisfactory cytoplasmic staining. The nuclei staining was optimal and distinct in RAT 1/220(0.5\%), MEM 2/220(0.9\%) and SAP 3/220(1.4\%) stained smears $(\mathrm{P}<0.001$; Figure 1; Table 2). The results further showed

Similarities in MEM and SAP procedures with $45 \%$ reduction in the diagnostic cost compared to RAT (Table 1). The LBC preparation for RAT revealed a distinct cell staining; MEM showed a less dark pyknotic nuclei staining and a better hue of color differentiation with lighter color shades of the cytoplasm. SAP showed a hue colouration of the cytoplasm appearing less tinted and faded with lesser pyknotic staining. RAT, MEM and SAP background appeared clear (Figure $1 \& 2$ ). 
Table 2: Comparison of Results (RAT, MEM, and SAP) for Physiognomy Analysis

\begin{tabular}{|l|l|l|l|l|}
\hline PROCF DURE & \multicolumn{2}{|l|}{ CY TOPLA SMIC STAIN } & \multicolumn{2}{l|}{ NUCLEI ST AIN } \\
\hline & Optimal & Sub-optimal & Optimal & Sub-optimal \\
\hline 220 RAT LBC preparation & 217 & 03 & 219 & 01 \\
\hline 220 MEM LBC preparation & 220 & 00 & 218 & 02 \\
\hline 220 SAP LBC preparation & 217 & 03 & 217 & 03 \\
\hline
\end{tabular}
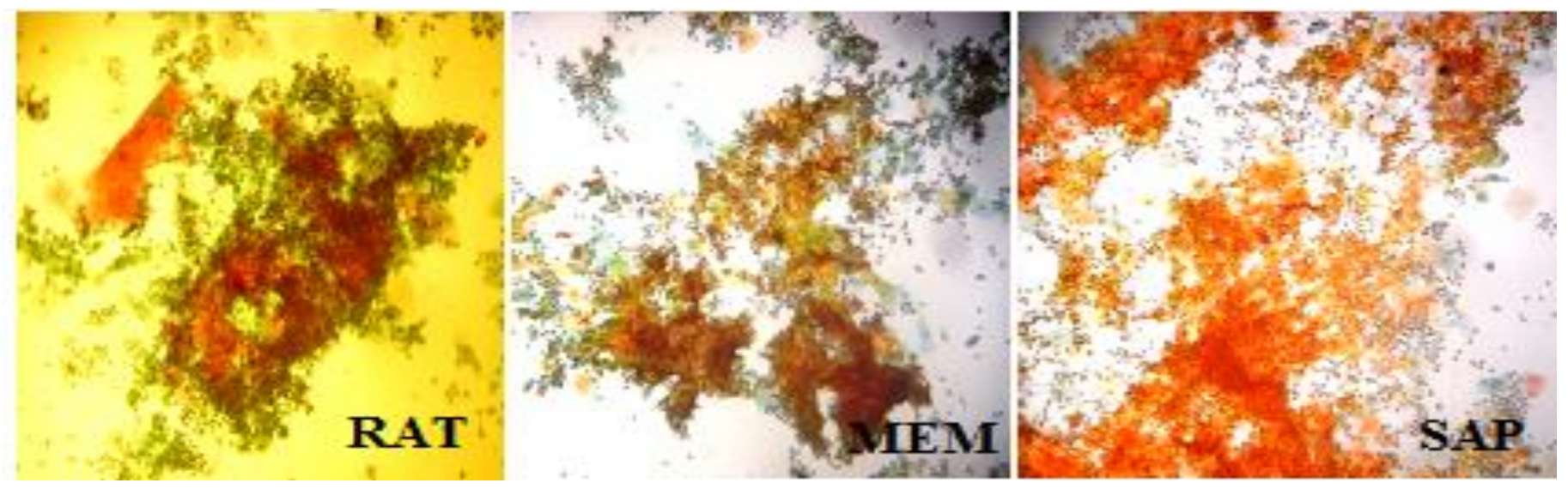

Figure 1: Showed staining uptake by RAT, MEM, and SAP at x10 modification.

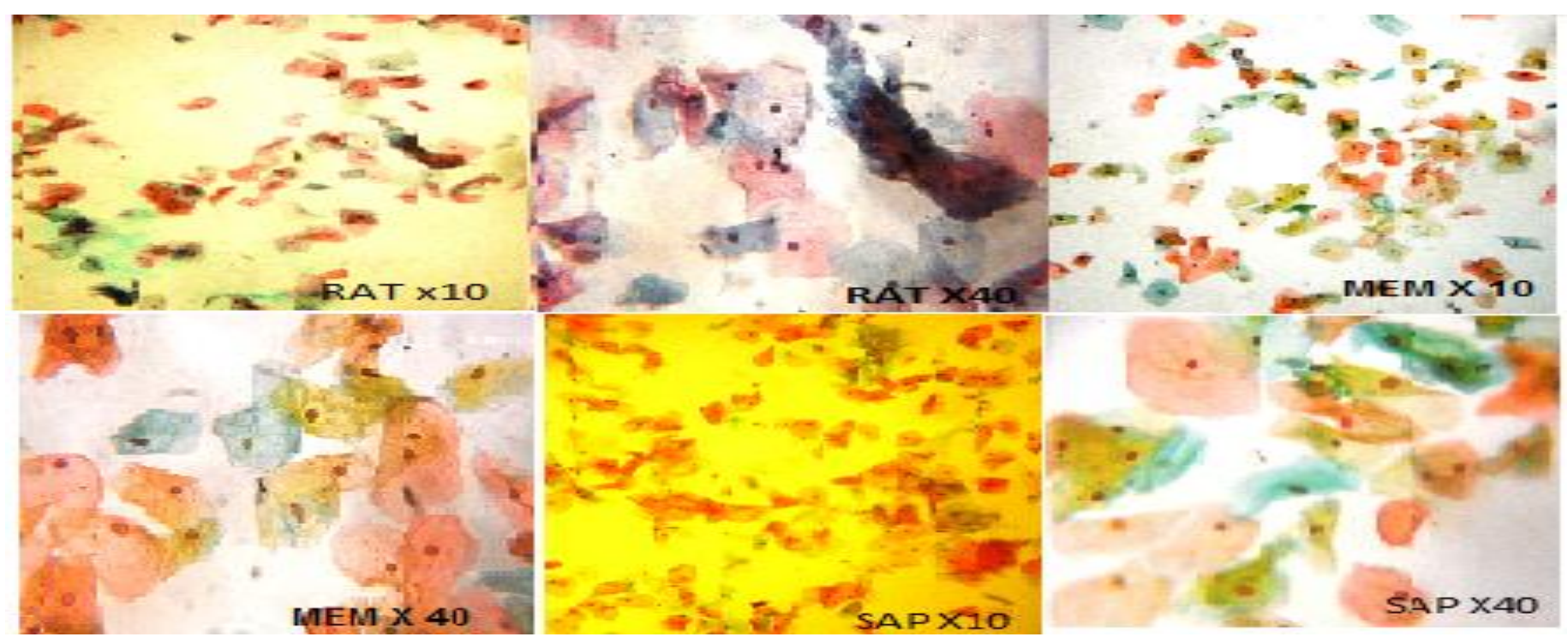

Figure 2: showed the characteristic features of RAT, MEM and SAP photomicrograph.

The RAT X10 \& X40 showed a normal smear stained with the modified Harris haematoxylin developed by Richard, (2013); otherwise known as the Richard Allan Thermo scientific method. MEM X10 \& X40 showed a normal smear stained with the modified emergency Pap staining method known as MEM by Odigie and Obaseki, (2014). SAP X10 \& X40 showed a normal smear stained with the re-modified version of MEM (the present study). 


\section{Discussion}

The most widely used staining procedure for cytological specimens is the Papanicolaou staining method. This technique is usually very colorful, which is the basis of contrast in stained smears. Colour contrast helps to aid identification of the nuclei, cytoplasm and background differentiation that results in a proper diagnosis ${ }^{[1,8-12]}$. In the first staining step, the nuclei are stained with a Haematoxylin solution (Nuclei are stained blue, dark violet to black). The second staining step is cytoplasmic staining by Orange staining solution, especially for a demonstration of mature and keratinized cells, and the target structures are stained orange in different intensities ${ }^{[10-13]}$. In the third staining step, the so-called Polychromatic solution is used (Azure Eosin). The Polychromatic solution is used for demonstration of differentiation of squamous cells e.g. cervical cancer and cycle diagnosis for examination under the microscope ${ }^{[1,5-}$ 9].

Modification of staining techniques was initially designed to save reagents, minimize the time required for staining and to improve the staining quality $[2,3,8]$. In the developed countries, modification of Pap staining technique was not geared towards saving cost, time and increases in the staining quality ${ }^{[8-11]}$ rather their major concerns focus on newer techniques that may stand the test of time and increase in turnaround time. However, the reception of any modified technique in the developing countries concentrate mainly on cost, which served as a prerogative compared to the quality of the stains. In the present study, the cost, quality of stains and turnaround time are the major focal point. Our ideas thus buttress the views by Alwahaibi et al. ${ }^{[8]}$ reporting that good cytology screening makes an accurate diagnosis devoid of mistakes. Our study aligns with earlier reports on the nuclear details, background, cell morphology, and overall staining being the essential features for a successful screening. These criteria have been considered in this study and are reported in previous studies ${ }^{[1,3,6,9-12]}$.

This study is consistent with some related works in the modification of Papanicolaou staining. Although, the ideas may have been obsolete in the likes of Rapid Papanicolaou stain by Sato et al. ${ }^{[6]}$; Rapid Economic Acetic-Acid Papanicolaou Stain REAP by Biswas et al. ${ }^{[7]}$ and the Diff-Quik Papanicolaou method by Dighe et al. ${ }^{[2]}$. Our study disagrees with the work by Richard ${ }^{[5]}$, in the time used for the nuclei and cytoplasmic staining (1 mins each), while the present study stained both features in $1 \mathrm{~min} 30 \mathrm{sec}$. The reasons for the disparity may not be readily ascertained. However, cutting cost and turn-around time, cost effectiveness is a leading role in any organized screening centers and hospitals across the country, especially in an economically depressed-developing country like Nigeria ${ }^{[1,14]}$. Our study thus agrees with Gupta et al. ${ }^{[3]}$; Belgaumi and Shetty ${ }^{[14]}$; in which modification of Pap staining was achieved with the aim of costcutting measure for resource-limited settings and may be suitable for use in Nigeria. Again, SAP agrees with MEM (45\% reduction in consumables), which has been published by Odigie and Obaseki ${ }^{[1]}$; and is also consistent with the report by Gupta et al. ${ }^{[3]}$, which favors minimum alcohol use but disagrees with Dighe et al. ${ }^{[2]}$; where $25 \%$ reduction was achieved. In respect to the turn-around time, SAP $(5 \pm 0.5)$ partially aligns with MEM $(7 \pm 0.5)$ by Odigie and Obaseki ${ }^{[1]}$ and the report by Choudhary et al. [4] but is dissimilar to RAT as reported by Richard ${ }^{[5]}$. In an earlier report, SAP staining quality agrees with MEM, which was well preserved even after some months of storage and is essential for smear review and retrospective studies ${ }^{[1,8,13,14]}$. Again, we stored the left over stains used for the three modified Papanicolaou methods and observed that the stains are well preserved in both MEM and SAP apart from being very active in smear staining even after one year (April 2016 to April 2017). A similar report by Alwahaibi et al. [8] has been reported in the comparison of Ultrafast Papanicolaou stain with the standard method in body fluids and fine needle aspiration specimens ${ }^{[9,11]}$. Nonetheless, in compares to RAT the stain was weak in the staining ability and uptake after one year of storage, which is comparable with the studies by Prachi and Surekha ${ }^{[9]}$; $\operatorname{Vari}^{[10]}$.

Summarily, Richard ${ }^{[5]}$ is an acidified formulation revealing specific nuclear staining and normal superficial and intermediate squamous cells with clusters of basal cells seen at both low power and high power magnification (Figure 2). The 
Modified Eosin-Azure acts as a cytoplasmic counterstain used for all cytology specimens including the LBC. Cellular results are consistent with basal or intermediate cells staining a bluegreen hue and surface cells staining a pink-red hue. The multicolor effect is due to selective dye uptake by various cells and their affinities for the specific dyes. The various formulations provide the end users with control over colour intensities and hues. EA 50 is used in conjunction with Orange G6 for the staining of gynaecological specimens. It will provide a very traditional staining result with natural staining hues and intensities. The liquid based cytology preparation reveals distinct cells and a clear background which has been reported ${ }^{[9,12]}$. However, MEM by Odigie and Obaseki ${ }^{[1]}$ staining reveals visible superficial and intermediate cells, which appear as distinct and well differentiated. The nucleus reveals less dark pyknotic staining, and the cytoplasm shows a better hue of colour differentiation with lighter colouration shades. These distinct characteristics are typical of a good Pap staining and have been reported ${ }^{[13,14]}$; while, the background appears clear and more revealing compared to RAT (Figure 2). Furthermore, the staining uptake for SAP reveals prominent superficial and intermediate cells which appear distinct and well differentiated. The hue colouration of the cytoplasm appeared less tinted and faded with lesser pyknotic staining while the background appears clear and better than RAT but less promising to MEM (Figure 2).

\section{Conclusion}

In the long run, SAP is time-saving and economically friendly than the other two methods (RAT and MEM). However, MEM remained an excellent qualitative staining method with a demonstrable turnaround time for all cytology specimens including LBC smears. Therefore, MEM is a better alternative for LBC samples and may be used in busy cytodiagnostic laboratories in Nigeria and the rest of the world.

\section{Acknowledgements}

Authors are thankful to Miss Atoigwe, B.E (Histopathology/ Cytopathology Unit, Dept of
Medical Laboratory Science, University of Benin); Mr. Odega, K.I. (Histology- Medical Laboratory Scientist) University of Benin Teaching Hospital (UBTH), and Mr. Erameh, T.O. (Igbinedion University, Okada) for their immense assistance towards the completion of this study.

\section{Disclosure}

Part of this paper has been presented in the $2^{\text {nd }}$ Edition of the University of Benin Academic Research Day (UBARD) as an extended abstract. Date: October 12 -14, 2016.

\section{Financial Support and Sponsorship}

Self-sponsored

\section{Conflicts of Interest}

No conflicts declared.

\section{References}

1. Odigie, B.E.; Obaseki, D.E. Conventional Pap Technique (CPT) In Comparison To the Modified Emergency Method (MEM) In Cytodiagnosis of Pap smear and Fine Needle Aspiration Cytology Specimens. Ann Biomed Sci., 2014; 13(1): 23-30.

2. Dighe, S.B.; Dulhan, A.; Saleem, P.; Chinoy, R. Papanicolaou Stain, Is it Economical to switch to rapid, economical, acetic acid, Papanicolaou stain? Acta Cytologica, 2006; 50(6):643-646. PMID: 17152276

3. Gupta, S; Chachra, K.L; Bhadola, P; Sodhani, P. Modified Papanicolaou staining protocol with minimum alcohol use: A cost-cutting measure for resource-limited settings. Cytopathology, 2010; 21:229-233. Doi:10.1111/j.13652303.2009.00699.x

4. Choudhary, P; Sudhamani, S; Pandit, A; Kiri, V. Comparison of modified Ultrafast Papanicolaou stain with the standard rapid Papanicolaou stain in cytology of various organs. J Cytol., 2012; 29:241-245. doi: 10.4103/0970-9371.103942

5. Richard-Allan Scientific. Thermo Scientific Rapid-Chrome Papanicolaou Staining Kit: 
Richard-Allan Scientific Subsidiary of Thermo Fisher Scientific 4481 Campus Drive, Kalamazoo, MI 49008; 2013. www.thermoscientific.com/pathology Retrieved: 2-12-2016.

6. Biswas, R. R.; Chandi, C.; Paral, R.D.; Biswas, S.C. Rapid Economic, Acetic Acid, Papanicolaou Stain (REAP) -Is it a suitable alternative to standard PAP stain? Al Ame en J Med Sci. 2008; 1(2):99-103. ajms.alameenmedical.org

7. Sato, M.; Taniguchi, E.; Kagiya, T.; Nunobiki, O.; Yang, Q.; Nakamura, M.; Nakamura, Y.; Mori, I.; Kakudo, K.A. Modified rapid Papanicolaou stain for imprint smear. Acta Cytologica, 2004; 48(3):461-462. PMID: 15192973.

8. Alwahaibi, N.Y; Alsubhi, M.S; Aldairi, N; Alshukaili, A; Bai, U.R. Comparison of Ultrafast Papanicolaou stain with the standard Papanicolaou stain in body fluids and fine needle aspiration specimens. $\boldsymbol{J}$ Lab Physicians, 2016; 8(1):19-24. doi: 10.4103/0974-2727

9. Prachi, S.; Surekha, U. A. Utility of Modified Ultrafast Papanicolaou Stain in Cytological Diagnosis. J Clin Diagn Res., 2017; 11(3): EC28-EC31. PMCID: PMC5427317. Doi: 10.7860/JCDR/2017/20882.9532

10. Vani, T.Y. The merits of rapid, economic acetic acid, Papanicolaou stain (REAP) over
Papanicolaou stain (PAP) technique in cervical cytology. Int J Res Med Sci., 2017;5:xxx-Xx. DOI: 10.18203/2320-6012.ijrms20173166

11. Patrikar, A.; Joshi, A.; Govardhan, V.; Dongre, T. Comparison of modified Ultrafast Papanicolaou stain with the standard Papanicolaou stain in cytology of various organs. IJSRP., 2016; 6(3):354-59. www.ijsrp.org

12. Izhar, S.; Kaur, R.; Masih, K. Efficiency of rapid economic acetic acid Papanicolaou stain in cervical smears as an alternative to conventional Papanicolaou stain. J Cytol., 2014; 3:31. PMCID: PMC4274527. Doi: 10.4103/09709371.145648.

13.Odigie, B.E.; Erameh, T.O.; Atoigwe, B.E.; Odega, K.I. Simplified Alternative method (SAP) for the Diagnosis of Liquid-based/ Cytology samples: A follow up to Odigie's Technique. $2^{\text {nd }}$ Edition, University of Benin Academic Research Day (UBARD), Book of Proceedings. University of Benin Press, Ekehuan Campus, Benin City, Nigeria, 2016; Pp. 39-42.

14.Belgaumi, U.I.; Shetty, P. Leishman Giemsa cocktail as a new, potentially useful cytological technique comparable to Papanicolaou staining for oral cancer diagnosis. J Cytol., 2013; 30:1822. DOI: $10.4103 / 0970-9371.107507$ 